ORIGINAL ARTICLE

\title{
Parathyroid invasion, nodal recurrence, and lung metastasis by papillary carcinoma of the thyroid
}

\author{
K Kakudo, W Tang, Y Ito, Y Nakamura, H Yasuoka, S Morita, A Miyauchi
}

J Clin Pathol 2004;57:245-249. doi: 10.1136/jcp.2003.012070

See end of article for authors' affiliations

.....................

Correspondence to:

Dr K Kakudo, Department

of Pathology, Wakayama Medical University, 811-1 Kimiidera, Wakayama City, Wakayama, 6418509 Japan; kakudo-k@ wakayama-med.ac.jp

Accepted for publication 11 September 2003

\begin{abstract}
Aims: Parathyroid invasion by papillary thyroid carcinoma (PTC) is found in a small proportion of surgical specimens, but the clinicopathological relevance of this phenomenon is not well understood. This study investigated the possible prognostic relevance of parathyroid invasion in PTC.

Method: Parathyroid involvement was seen in 14 patients with PTC, and the clinicopathological characteristics and follow up data of these patients were analysed and compared with 164 patients without parathyroid involvement, in whom histological parathyroid examination had been undertaken, and 177 other patients without parathyroid examination (341 patients without parathyroid involvement in total).

Results: Parathyroid invasion was found in older patients and there were more male patients in this group than in those without parathyroid invasion. These patients had more extrathyroid extension and were frequently in an advanced stage of disease. Lung metastasis was seen in two of the 14 patients, which was significantly more than that seen in control cases. Moreover, male patients with parathyroid invasion and those who were older than 55 years had reduced disease free survival compared with those without parathyroid invasion.

Conclusion: Parathyroid invasion seen in thyroid carcinoma may be an important histological feature indicating a greater chance of nodal recurrence and lung metastasis.
\end{abstract}

$\mathrm{T}$ here are four parathyroid glands, which are located (usually two each) on the lateral sides of the thyroid gland. ${ }^{1}$ They are often dissected with the thyroid during surgery for thyroid carcinoma. To prevent postoperative hypoparathyroidism, a meticulous search for parathyroid glands is carried out at surgery. However, owing to their small size, they are often overlooked after surgery, and even at the histopathological examination for cancer extension they may be dissected together with the thyroid. Therefore, the clinical relevance of parathyroid involvement by papillary thyroid carcinoma (PTC) and its incidence have not yet been well documented. ${ }^{2-4}$ In our previous study, we described parathyroid involvement by PTC in $2.2 \%$ of cases. ${ }^{5}$

"The parathyroid glands are often dissected with the thyroid during surgery for thyroid carcinoma"

In our present study, we investigated the prognostic relevance of parathyroid involvement by PTC in a series of patients whose follow up data were available.

\section{MATERIALS AND METHODS}

Three hundred and seventy patients with PTC, who were surgically treated between February 1992 and June 1993 at Kuma Hospital, Kobe, Japan, were reviewed. A variety of surgical procedures were carried out, namely: enuclation, lobectomy, lobectomy with ischmectomy, ischmectomy, subtotal thyroidectomy, near total thyroidectomy, and total thyroidectomy. Fifteen patients whose follow up data were not available were excluded. In these 15 patients, there was one with parathyroid involvement, who was excluded from the study. During surgery, parathyroid glands were identified in the remaining 355 cases. However, in 177 cases they were not removed so that no histological slides of these parathyroid glands were available. In the other 178 cases, at least one parathyroid gland was dissected separately or together with the thyroid gland, and these parathyroids were examined histologically. Fourteen patients were found to have cancer deposits in the parathyroid glands among the 178 PTCs. Clinicopathological data including age, sex, multiplicity, size of the primary tumour, extrathyroid extension, nodal metastasis, and tumour stage were obtained from the clinical files of the Kuma Hospital, Japan. Histopathological review was performed by two of the authors (KK and WT). Multiplicity was represented by two or more lesions of papillary carcinoma occurring separately within the ipsilateral or contralateral lobe, whereas cancer nests within the lymphatic channels were regarded as intrathyroidal metastasis. Extrathyroid extension and nodal metastasis were confirmed histologically. Extrathyroid extension included invasion into fat, muscle, nerve fibres, oesophagus, jugular vein, and trachea beyond the thyroid capsule. Tumour stage grouping was carried out based on the pTNM pathological classification referred to as the UICC TNM classification. ${ }^{6}$ All 355 cases were diagnosed as differentiated PTC. Other histological types of thyroid cancer were excluded from our study, such as follicular carcinoma, medullary carcinoma, anaplastic carcinoma, or malignant lymphoma.

Follow up data were available for all 355 patients and the average follow up period was 87.6 months (range, 2-128). Recurrence in the residual thyroid gland or in a lymph node was confirmed histologically. Distant metastasis at diagnosis or during the follow up period was identified by means of computed tomography, radioiodine scintigraphy, and routine chest radiography. The disease free survival period was calculated from the time of primary surgery to the final contact day or the day that recurrence was identified.

Abbreviation: PTC, papillary thyroid carcinoma 


\section{Statistical analysis}

Time independent categorical data were evaluated using the $\chi^{2}$ test or the Student's $t$ test. Survival analysis was performed with StatView-J 5.0 statistical software packages (SAS Institute Inc, Cary, North Carolina, USA). For univariate analysis of time dependent variables, the KaplanMeier method and the log rank test were used to determine significant prognostic factors. The differences observed were assumed to be significant if the probability was $<0.05$.

\section{RESULTS}

Parathyroid involvement was found in $14(7.9 \%)$ of 178 cases of PTC with histological parathyroid examination. When the incidence was calculated in all 355 cases, it became 14 of 355 (3.9\%). In 14 cases with parathyroid involvement, one parathyroid gland was examined histologically in 10 cases, two glands in three cases, and three glands in one case. Only one parathyroid gland was found to be involved by PTC in each of the 14 cases. The parathyroid glands involved by PTC were located outside the thyroid gland in all 14 cases, and no intrathyroidal parathyroid cases were found. In 10 of the 14 cases, the parathyroid gland was directly invaded by PTC, and in three other cases the PTC extended into the parathyroid gland with an intervening fibrous capsule. Cancer deposits in the parathyroid gland with no continuity from the main tumour was seen in only one case in our series. ${ }^{5}$

The average age of the 14 patients with PTC with parathyroid involvement was 60.4 years, which was older than that of the 164 patients without parathyroid involvement (49.8 years old) and that of the total 341 patients without parathyroid involvement (49.1 years old) (table 1). The difference was significant $(p=0.0030)$. Of the 14 patients with parathyroid involvement, four were men (four of $14 ; 28.8 \%)$. The male ratio was higher than that seen in the 164 control cases ( 10 of $164 ; 6.1 \%$ ) and the total 341 cases $(17 / 324 ; 5.2 \%)$. In addition to parathyroid involvement, parathyroid invasion beyond the thyroid capsule and into fat and/or muscle was seen in 13 of the 14 patients. No other adjacent tissue structures, such as the trachea, oesophagus, jugular vein, etc were involved in these 14 patients. The incidence of parathyroid invasion was significantly higher than that seen in the 164 control cases $(p=0.0156)$ and the total 341 cases $(p=0.0010)$. Multiplicity of PTC was seen in six patients. The average diameter of the tumours invading the parathyroid in the 14 cases was $2.5 \mathrm{~cm}$ (range, 0.5$5.8 \mathrm{~cm}$ ). Thirteen of the 14 cases showed metastasis in one or more regional lymph node. Based on the tumour size, extrathyroid extension, and/or metastasis, 12 of the 14 cases were classified into stage III/IV, one case belonged to stage II, and another one belonged to stage I. Advanced stages were seen more frequently in those PTCs with parathyroid involvement than in the 164 control cases or the total 341 cases. However, there were no significant differences for tumour size, multiplicity, incidence of lymph node metastasis, and recurrence between those PTCs with parathyroid involvement and those without parathyroid involvement.

Follow up data were analysed in all the 178 patients with histological parathyroid examination in our series. The follow up period ranged from two months to 128 months and the mean follow up period was 87 months, with 135 patients having more than five years of follow up. Two of the 14 patients with PTC and parathyroid involvement had a recurrence, whereas recurrence occurred in 10 of the 164 control patients and 22 of the 341 total negative patients. The frequency of recurrence was not significantly different between the three groups $(p=0.2409)$. Lung metastasis was seen in two patients with PTC and parathyroid involvement, but in only one of the 164 control patients with PTC and in four of the 341 total patients. The difference between the occurrence of lung metastasis in the patients with parathyroid involvement and the 164 control patients was significant $(p=0.0001)$.

The disease free survival rate of the 14 patients with parathyroid involvement was $80.8 \%$, which was less than

Table 1 Comparison of papillary thyroid carcinomas with parathyroid involvement and those without parathyroid involvement

\begin{tabular}{|c|c|c|c|c|c|}
\hline & \multicolumn{5}{|c|}{ Parathyroid involvement } \\
\hline & \multirow[b]{2}{*}{ Yes $(n 1=14)$} & \multicolumn{4}{|l|}{ No } \\
\hline & & With histology (n2 = 164) & $p$ Value $(n 1 \vee n 2)$ & Total number $(n 3=341)$ & $p$ Value $(n 1 \vee n 3)$ \\
\hline Age (mean) & 60.4 & 49.8 & 0.0030 & 49.1 & 0.0017 \\
\hline \multicolumn{6}{|l|}{ Sex } \\
\hline Female & 10 & 154 & 0.0027 & 324 & 0.0002 \\
\hline Male & 4 & 10 & & 17 & \\
\hline Tumour size $(\mathrm{cm})$ & 2.5 & 2.0 & 0.1661 & 1.9 & 0.2713 \\
\hline \multicolumn{6}{|l|}{ Multiplicity } \\
\hline Single & 8 & 123 & 0.1457 & 244 & 0.2442 \\
\hline Multiple & 6 & 41 & & 97 & \\
\hline \multicolumn{6}{|l|}{ Extrathyroid invasion } \\
\hline No & 1 & 75 & 0.0051 & 178 & 0.0010 \\
\hline Yes & 13 & 89 & & 163 & \\
\hline \multicolumn{6}{|l|}{ Nodal metastasis* } \\
\hline No & 1 & 45 & 0.0819 & 84 & 0.0839 \\
\hline Yes & 13 & 112 & & 214 & \\
\hline \multicolumn{6}{|l|}{ Lung metastasis } \\
\hline No & 12 & 163 & 0.0001 & 337 & 0.0002 \\
\hline \multirow{2}{*}{\multicolumn{6}{|c|}{ Stage $^{*}$}} \\
\hline & & & & & \\
\hline |/II & 2 & 87 & 0.0032 & 167 & 0.0022 \\
\hline III/IV & 12 & 70 & & 131 & \\
\hline \multicolumn{6}{|l|}{ Recurrence } \\
\hline No & 12 & 154 & 0.2409 & 319 & 0.2525 \\
\hline Yes & 2 & 10 & & 22 & \\
\hline \multicolumn{6}{|l|}{ Surgery } \\
\hline Lobectomy† & 3 & 69 & 0.1309 & 147 & 0.1075 \\
\hline Thyroidectomy $\ddagger$ & 11 & 95 & & 194 & \\
\hline
\end{tabular}




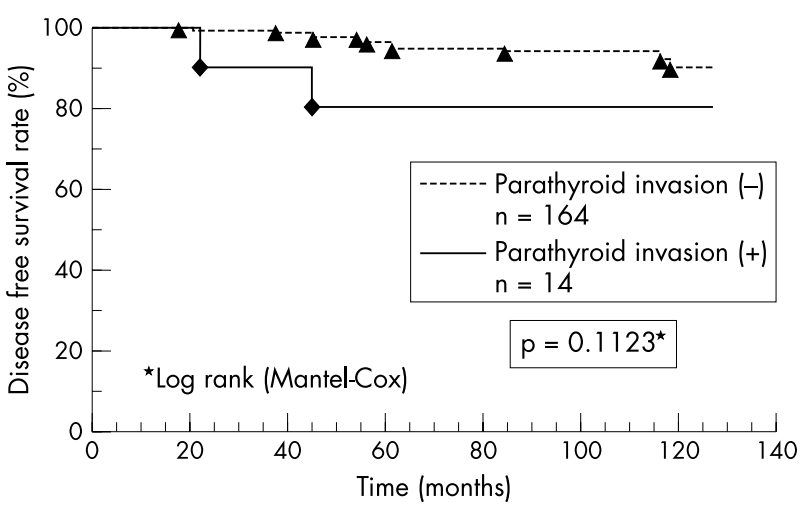

Figure 1 Disease free survival curve (Kaplan-Meier method) of patients with papillary thyroid carcinoma, with and without parathyroid involvement. The 10 year disease free survival rate of patients with parathyroid invasion was $80.8 \%$, which was not significantly different from those without parathyroid invasion $(90.1 \%)$.

that of the 164 patients with histological parathyroid examination and the total 341 patients with PTC but no parathyroid involvement (table 1 ), although the difference was not significant ( $\mathrm{p}=0.1123$; fig 1$)$.

Parathyroid involvement was seen in nine patients older than 55 years, and their disease free survival rate was significantly lower than the 64 patients without parathyroid involvement who were older than 55 (fig 2; p = 0.0027). Furthermore, when the patients were divided into four groups based on sex and parathyroid involvement, male patients with parathyroid involvement also showed lower disease free survival (fig 3). However, no significant difference for disease free survival was found between PTCs with or without parathyroid involvement when other clinicopathological factors, such as nodal status, extrathyroid extension, or pathological stage, were incorporated.

Extrathyroid invasion was seen in 102 patients with PTC13 cases with parathyroid involvement and 89 without parathyroid involvement. Table 2 shows a comparison of the clinicopathological parameters in the two groups of PTCs with extrathyroid invasion. Parathyroid involvement was frequently seen in the PTCs of older male patients. Among the 13 extrathyroid invasion positive patients with PTC and parathyroid invasion, two had lung metastases, whereas only one of the 89 extrathyroid invasion positive patients without parathyroid invasion had lung metastases $(p=0.0045)$, although there were also more stage III cases among the parathyroid invasion positive PTCs than among the parathyroid invasion negative PTCs (11 of $13 v 48$ of 89;

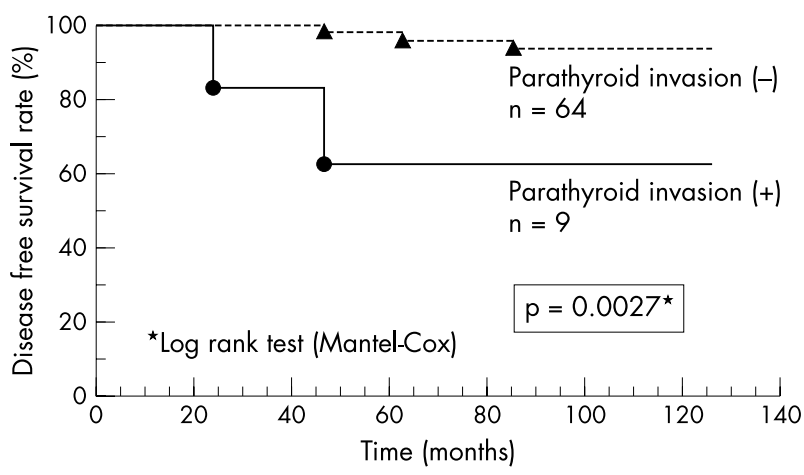

Figure 2 Parathyroid involvement and disease free survival of patients greater than 55 years old (Kaplan-Meier method). The disease free survival rate of patients with parathyroid invasion was significantly lower than those without parathyroid invasion ( $p=0.0027$ ).

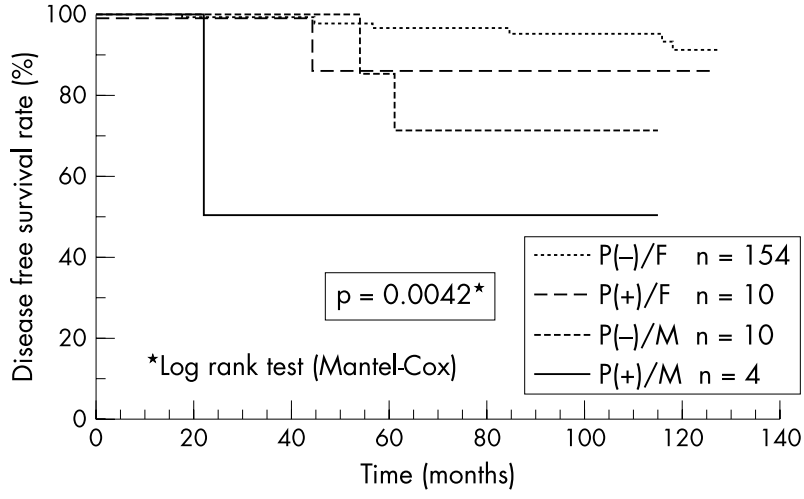

Figure 3 Parathyroid involvement, sex, and disease free survival of patients with papillary thyroid carcinoma (PTC) (Kaplan-Meier method). PTCs were divided into four groups according to parathyroid invasion and sex. Male patients with parathyroid invasion had the lowest disease free survival rate. The differences among the four groups were significant $(p=0.0042)$. F, female; $M$, male; $P(-)$, parathyroid involvement negative; $\mathrm{P}(+)$, parathyroid involvement positive.

$\mathrm{p}=0.0441)$. When the comparison was made in only stage III cases (two of $11 v$ one of 48), the difference was still significant $(\mathrm{p}=0.0284)$.

Venous invasion was seen more in PTCs that were parathyroid invasion positive (three of $13 v$ six of 89), although the difference was only of borderline significance $(\mathrm{p}=0.0524)$. We also noted a close relation between venous invasion and lung metastasis, because venous invasion was positive in two of three patients with PTC and lung metastasis, and these two patients also exhibited parathyroid invasion. No statistical analysis was carried out because of the small numbers of patients.

\section{DISCUSSION}

In our present study, we found parathyroid involvement in $7.9 \%$ of patients with PTC, whose parathyroid glands were histologically identified, and the rate was 3.9\% of all 355 PTCs. The frequency was slightly higher than that seen in our previous study. ${ }^{5}$ This figure is probably the most accurate one because dissected parathyroid glands were identified histologically in all of the 178 PTCs, providing a greater chance of identifying parathyroid gland involvement.

Our results suggest that parathyroid involvement by PTC is not a rare phenomenon. However, to the best of our knowledge, there have been no publications describing the clinicopathological relevance of parathyroid invasion by PTC in detail. ${ }^{2-4}$

Among various clinicopathological factors, extrathyroid extension, tumour infiltration into the surrounding tissues beyond the thyroid capsule, is one of the well established predictors of a poor prognosis in patients with thyroid carcinoma. $^{8-12}$ As has been mentioned in many previous reports, the adjacent tissue structures potentially invaded by thyroid carcinoma include: fat, muscle, the oesophagus, the trachea, the vagus nerves, the thymus, the carotid artery, and the jugular vein, but involvement of the parathyroid gland has not described in the major textbooks or publications on PTC. $^{8-12}$ In our previous study, parathyroid gland invasion occurred in $2.2 \%$ of surgically treated patients. ${ }^{5}$ Although this phenomenon was often accompanied by fat and/or muscle invasion, in our present study we also found that patients with parathyroid involvement by PTC have a greater chance of lung metastasis. In addition, recurrence frequently occurred in patients with parathyroid involvement, who had a relatively low disease free survival compared with those without parathyroid involvement, although the differences 


\begin{tabular}{|c|c|c|c|}
\hline & \multicolumn{2}{|c|}{ Parathyroid involvement } & \multirow[b]{2}{*}{ p Value } \\
\hline & Positive ( $n=13$ ) & Negative $(n=89)$ & \\
\hline Mean age (years) & 60.0 & 52.8 & 0.0465 \\
\hline \multicolumn{4}{|l|}{ Sex } \\
\hline Male & 3 & 5 & 0.0287 \\
\hline Female & 10 & 84 & \\
\hline Tumour size $(\mathrm{cm})$ & 2.6 & 2.2 & 0.3043 \\
\hline \multicolumn{4}{|l|}{ Multiplicity } \\
\hline Single & 6 & 18 & 0.0395 \\
\hline Multiple & 7 & 71 & \\
\hline \multicolumn{4}{|l|}{ Nodal metastasis* } \\
\hline Negative & 1 & 16 & 0.3381 \\
\hline Positive & 12 & 71 & \\
\hline \multicolumn{4}{|l|}{ Lung metastasis } \\
\hline Negative & 11 & 88 & 0.0045 \\
\hline Positive & 2 & 1 & \\
\hline \multicolumn{4}{|l|}{ Vascular invasion } \\
\hline Negative & 10 & 83 & 0.0524 \\
\hline Positive & 3 & 6 & \\
\hline \multicolumn{4}{|l|}{ Stage ${ }^{*}$} \\
\hline$|/| \mid$ & 2 & 39 & 0.0441 \\
\hline III/IV & 11 & 48 & \\
\hline \multicolumn{4}{|l|}{ Recurrence } \\
\hline Negative & 11 & 83 & 0.2789 \\
\hline Positive & 2 & 6 & \\
\hline \multicolumn{4}{|l|}{ Surgery } \\
\hline Lobectomy & 3 & 39 & 0.1557 \\
\hline Thyroidectomy & 10 & 50 & \\
\hline
\end{tabular}

between the two groups were not significant, which may partly result from the small sample size of PTCs with parathyroid involvement ( 14 cases). When comparing those patients older than 55 years, we also noted with interest that parathyroid involvement was a significant indicator of disease free survival. The other clinical parameters of the 14 patients that were significantly different from the controls were that the patients were older, there was a relatively higher proportion of men, and the patients had a more advanced stage of the disease. These parameters were also reported as predictors of poor prognosis in PTC. ${ }^{8-11}$ Older age has been reported as a predictor of worse prognosis, although an exact cutoff point was not clearly identified in a large scale study. ${ }^{13}$ In our study, we found that disease recurred more frequently in patients older than 55 years, and that these patients had a significantly lower disease free survival rate, whereas if the cutoff point was 45 years of age the difference was not significant.

"Our results suggest that parathyroid involvement by papillary thyroid carcinoma is not a rare phenomenon"

Parathyroid glands are very small organs and are located outside or close to the thyroid gland capsule. ${ }^{14-16}$ Their local circulation, particularly lymphatic drainage, has not been well established. Using a lymphatic endothelium specific marker (monoclonal antibody against flt4), ${ }^{17}{ }^{18}$ no lymphatic channels were identified immunohistochemically in the parathyroid glands involved by PTC (data not shown), and many capillaries and small blood vessels were positively stained by antibodies to CD34 and smooth muscle actin. The provision of a rich blood supply in parathyroid glands with PTC invasion may be related to the higher rate of lung metastasis in those patients.

In addition, our study found that the incidence of parathyroid involvement by PTC could be as high as $7.9 \%$. At thyroid surgery, parathyroid transplantation is often carried out to prevent hypoparathyroidism. ${ }^{19-22}$ Parathyroid tissue is confirmed histologically by a pathologist during surgery using cryostat sectioning, but the identification of invasive PTC in parathyroid tissue may be difficult in such sections, and is often ignored. To avoid the transplantation of PTC together with parathyroid tissue, pathologists should be aware that up to $7.9 \%$ of parathyroids may be involved by PTC. Thus, parathyroid tissue for autotransplantation should be selected from the contralateral side (which has never been seen to be involved by cancer) instead of the ipsilateral side, which has a greater chance of cancer invasion.

In conclusion, in our study parathyroid involvement was seen in a group of patients with PTC who were older, in a more advanced stage of disease, and had a greater incidence of lung metastasis compared with controls; in addition there was a relatively higher proportion of men in this group. Although the cases with parathyroid involvement were small

\section{Take home messages}

- Parathyroid involvement was seen in $7.9 \%$ of papillary thyroid carcinomas (PTCs) with histological parathyroid examination and $3.9 \%$ of all PTCs

- Parathyroid involvement was seen in a group of patients with a relatively higher proportion of men, who were older, in a more advanced stage of disease, and had a greater incidence of lung metastasis compared with controls

- Parathyroid involvement correlated with shorter disease free survival

- Parathyroid invasion in thyroid carcinoma is an important histopathological parameter indicating an aggressive course of disease 
in number, this phenomenon correlated with shorter disease free survival. Therefore, parathyroid invasion in thyroid carcinoma is one of the important histopathological parameters indicating an aggressive course.

\section{Authors' affiliations}

K Kakudo, W Tang, Y Nakamura, H Yasuoka, Department of

Pathology, Wakayama Medical University, $811-1$ Kimiidera,

Wakayama City, Wakayama, 641-8509, Japan

Y Ito, S Morita, A Miyauchi, Kuma Hospital, Kobe 650-001 1, Japan

\section{REFERENCES}

1 Mansberger AR, Wei JP. Surgical embryology and anatomy of the thyroid and parathyroid glands. Surg Clin North Am 1993;73:727-46.

2 Damjanov I, Linder J. Anderson's pathology, 10th ed. St Louis, MO: Mosby, 1996.

3 Rosai J. Ackerman's surgical pathology, 8th ed. St Louis, MO: Mosby, 1996.

4 Sternberg SS, Antonioli DA, Carter D, et al. Diagnostic surgical pathology, 3rd ed. Philadelphia, PA: Lippincott Williams \& Wikins, 1999.

5 Tang W, Kakudo K, Nakamura Y, et al. Parathyroid gland involvement by papillary carcinoma of the thyroid gland. Arch Pathol Lab Med 2002; 126:1511-14

6 Sobin LH, Wittekind C, eds. UICC: TNM classification of malignant tumours, 5th ed. New York, NY: Wiley-Liss, Inc, 1997.

7 Mori I, Miyauchi A, KumaSeiii, et al. Thyroid nodular lesion-analysis of cancer risk based on Kuma Hospital experience. Pathol Int 2003;53:579-83.

8 Simpson WT, McKinny SE, Carruthers JS, et al. Papillary and follicular thyroid cancer. Prognostic factors in 1,578 patients. Am J Med 1987;83:479-88.

9 Akslen LA, Myking AO, Salvesen H, et al. Prognostic importance of various clinicopathological features in papillary thyroid carcinoma. Eur J Cancer 1992;29A:44-51.
10 Carcangiu ML, Zampi G, Pupi A, et al. Papillary carcinoma of the thyroid. A clinicopathologic study of 241 cases treated at university of Florence, Italy. Cancer 1985;55:805-28.

11 DeGroot L, Kaplan EL, McCormick M, et al. Natural history, treatment, and course of papillary thyroid carcinoma. J Clin Endocrinol Metab 1990;71:414-24.

12 Nishida T, Nakao K, Hashimoto T. Local control in differentiated thyroid carcinoma with extrathyroidal invasion. Am J Surg 2000;179:86-91.

13 Gilliland FD, Hunt WC, Morris DM, et al. Prognostic factors for thyroid carcinoma. A population-based study of 15,698 cases from the surveillance, epidemiology and end results (SEER) program 1973-1991. Cancer 1997;79:564-73.

14 Iwasaki A, Shan L, Kawano I, et al. Quantitative analysis of stromal fat content of human parathyroid glands associated with thyroid diseases using computer image analysis. Pathol Int 1995;45:483-86.

15 Shan L, Iwasaki A, Utsunomiya $H$, et al. Immunoreactive characteristics and classification of hyperparathyroidism. Endocr Pathol 1995;6:145-52.

16 Shan L, Nakamura M, Nakamura Y, et al. Comparative analysis of clonality and pathology in primary and secondary hyperparathyroidism. Virchows Arch 1997; 430:247-51.

17 Kaipainen A, Korhonen J, Mustonen T, et al. Expression of the fms-like tyrosine kinase 4 gene becomes restricted to lymphatic endothelium during development. Proc Natl Acad Sci U S A 1995:92:3566-70.

18 Nakamura Y, Yasuoka H, Tsujimoto $M$, et al. Clinicopathological significance of vascular endothelial growth factor-C in breast carcinoma with long-term follow-up. Mod Pathol 2003;16:309-14.

19 Sturniolo G, Lo Schiavo MG, Tonante A, et al. Hypocalcemia and hypoparathyroidism after total thyroidectomy: a clinical biological study and surgical considerations. Int J Surg Investig 2000;2:99-105.

20 Thomusch O, Machens A, Sekulla C, et al. The impact of surgical technique on postoperative hypoparathyroidism in bilateral thyroid surgery: a multivariate analysis of 5846 consecutive patients. Surgery 2003;133:180-5.

21 Lo CY. Parathyroid autotransplantation during thyroidectomy. ANZ J Surg 2002;72:902-7

22 D'Avanzo A, Parangi S, Morita E, et al. Hyperparathyroidism after thyroid surgery and autotransplantation of histologically normal parathyroid glands. J Am Coll Surg 2000;190:546-52. 